\title{
Surgical Approach Including Hyperthermic Intraperitoneal Chemotherapy (HIPEC) in Patients with Peritoneal Metastasis
}

\author{
Gabriel Glockzin $^{\mathrm{a}}$ Pompiliu Piso $^{\mathrm{b}}$ Hans J. Schlitt ${ }^{\mathrm{a}}$ \\ a Department of Surgery, University Medical Center Regensburg, \\ ${ }^{\mathrm{b}}$ Department of Surgery, St. John of God Hospital Regensburg, Regensburg, Germany
}

Keywords

Cytoreductive surgery · HIPEC - Peritoneal metastasis

\section{Summary}

Background: Peritoneal metastasis arising from colorectal cancer, appendiceal cancer, gastric cancer and gynecologic malignancies, or primary peritoneal surface malignancies such as peritoneal mesothelioma and primary peritoneal adenocarcinoma may be efficiently treated by cytoreductive surgery (CRS) and hyperthermic intraperitoneal chemotherapy (HIPEC) in selected patients. Method: CRS is based on the technique of parietal and visceral peritonectomy and consists of multiple surgical procedures. HIPEC combines high local doses of cytostatics with the additional cytotoxic effects of hyperthermia. Results: The goal of CRS is to achieve a complete macroscopic cytoreduction (CC-0/1) as a precondition for consecutive HIPEC that should destroy residual tumor cells within the abdominal cavity. Conclusion: CRS and HIPEC can be performed with acceptable morbidity and low mortality in specialized centers. However, due to long learning curves, consistent surgical training is strongly recommended.

\section{Introduction}

Peritoneal metastasis is a common sign of advanced tumor stage, disease progression, or recurrence in numerous tumor entities of gastrointestinal or gynecological origin. Moreover, there are primary peritoneal malignancies such as malignant
Schlüsselwörter

Zytoreduktive Chirurgie · HIPEC · Peritonealkarzinose

\section{Zusammenfassung}

Hintergrund: Die zytoreduktive Chirurgie (CRS) und hypertherme intraperitoneale Chemotherapie (HIPEC) stellen eine effiziente Behandlungsoption für selektionierte Patienten mit Peritonealkarzinose bei diversen gastrointestinalen Karzinomen und Ovarialkarzinom bzw. malignem peritonealen Mesotheliom oder primärem Adenokarzinom des Peritoneums dar. Methode: Die CRS basiert auf der Technik der parietalen und viszeralen Peritonektomie und beinhaltet multiple Prozeduren. Die HIPEC kombiniert hohe lokale Zytostatikadosen mit den additiven zytotoxischen Effekten der Hyperthermie. Ergebnisse: Ziel der CRS ist die komplette makroskopische Zytoreduktion (CC-0/1) als Voraussetzung für die Durchführung einer intraoperativen HIPEC, die intraabdominell verbliebene Tumorzellen zerstören soll. Schlussfolgerung: CRS und HIPEC können in spezialisierten Zentren mit akzeptabler Morbidität und niedriger Mortalität durchgeführt werden. Aufgrund der langen Lernkurve sind jedoch eine strukturierte Ausbildung und ein adäquates chirurgisches Training dringend zu empfehlen.

peritoneal mesothelioma or primary peritoneal carcinoma. Peritoneal tumor dissemination is still often associated with poor prognosis $[1,2]$. The concept of cytoreductive surgery (CRS) and hyperthermic intraperitoneal chemotherapy (HIPEC) as first described by Sugarbaker et al. [3] in 1989 provides an additive promising treatment option for a se-

\section{KARGER \\ Fax +497614520714}

Information@Karger.com

www.karger.com (c) 2013 S. Karger GmbH, Freiburg

1662-6664/13/0294-0220\$38.00/0

Accessible online at:

www.karger.com/vim 
lected group of patients with peritoneal tumor dissemination $[3,4]$. Although there are only few prospective randomized trials, several studies and retrospective analyses could show that CRS and HIPEC as an integrative part of an interdisciplinary treatment concept may improve the oncological outcome of selected patients with peritoneal metastasis $[1,5]$. However, the multimodal treatment is associated with significant adverse effects. By analyzing the data from 24 international specialized centers, Chua et al. [6] reported a mean grade III/ IV morbidity and mortality rate of 28.8 and $2.9 \%$, respectively. The following article summarizes the surgical approach, intraoperative decision making, and selected technical aspects regarding peritonectomy procedures and HIPEC.

\section{Cytoreductive Surgery}

CRS consists of numerous surgical procedures depending on the extent of peritoneal tumor manifestation. Surgery may include parietal and visceral peritonectomy, greater and lesser omentectomy, and single organ as well as multivisceral resection. Moran et al. [7] described the following five basic procedures: i) right hemicolectomy, greater and lesser omentectomy, and splenectomy; ii) right and left diaphragmatic peritonectomy; iii) cholecystectomy and resection of Glisson's capsule; iv) (partial) gastrectomy; and v) anterior rectal resection, pelvic peritonectomy, hysterectomy, and ovariectomy. The goal of CRS is to remove all visible intraperitoneal tumor nodules. The completeness of cytoreduction can be determined by the completeness of the cytoreduction score. CC-0 indicates no visible residual tumor and CC-1 residual tumor nodules $\leq 2.5 \mathrm{~mm}$. CC-2 and CC-3 indicate residual tumor nodules between $2.5 \mathrm{~mm}$ and $2.5 \mathrm{~cm}$ and $>2.5 \mathrm{~cm}$, respectively [8]. Nevertheless, as single tumor nodules $\leq 2.5 \mathrm{~mm}$ within the abdominal cavity may remain unnoticed during surgery, especially in patients with a high tumor load, a complete macroscopic cytoreduction as a precondition for HIPEC is classified as CC- $0 / 1$ by most surgical oncologists.

\section{Surgical Technique}

Peritonectomy procedures qualify for numerous surgical techniques and the use of multiple different instruments. At least, monopolar electrosurgery and bipolar coagulation forceps are recommended. Additional instruments such as bipolar scissors, argon beam, or tissue sealing devices may be helpful. Preparation may be performed by sharp and blunt dissection depending on the procedure, the surgeon's preferred technique, and the individual intraoperative findings. The technique is based on the principle of traction and countertraction that allows for parietal as well as visceral peritoneal stripping [9]. In contrast to the standard procedure, some organ resections such as splenectomy, rectal resection, or hysterectomy have to be performed via an extraperitoneal approach. Consistent surgical tutoring by specialists and training in specialized centers for peritoneal surface malignancies is strongly recommended for CRS. Under these conditions, a learning curve ranging from 60 to 158 patients has been reported for CRS (and HIPEC) to achieve acceptable safety and oncologic radicality [10].

\section{Surgical Approach}

The patient is placed in the modified lithotomy position that should be accurately controlled before surgery to avoid postoperative compartment syndrome of the lower leg with consecutive skin, muscle, and/or nerve injury. A complete median laparotomy is performed with a median skin incision from the xiphoid to the symphysis. Omphalectomy is not mandatory in all patients and should be performed depending on the primary tumor, tumor load, and dissemination pattern. However, in patients with high-volume pseudomyxoma peritonei as well as patients with periumbilical scars after previous resection or exploration of malignant mesothelioma, omphalectomy should be routinely performed. Especially in patients with malignant peritoneal mesothelioma, pseudomyxoma peritonei, and high tumor load, the integrity of the parietal peritoneum should be preserved to allow for easier extraperitoneal preparation and parietal peritonectomy. Skin sutures that are fixed on the frame of the retraction system and holding tension by clamps may facilitate this first step of surgical preparation. Following its exposure, the parietal peritoneum is incised and the abdominal cavity is accurately explored.

The extent of abdominal tumor dissemination is determined using the Peritoneal Cancer Index (PCI), a combined numerical score of lesion size (LS-0 to LS-3) and tumor location (region $0-12$ ) that ranges from 0 to $39[8,11]$. The abdominal exploration plays a pivotal role in intraoperative decision making regarding the indication for CRS and HIPEC. Whereas there are no PCI-associated limitations in patients with pseudomyxoma peritonei, based on the survival data in patients with peritoneal disease from colorectal cancer the intraoperative PCI should be lower than 20 and in patients with gastric cancer at least lower than 10. Esquivel et al. [12, 13] have shown that preoperative CT scans often underestimate the intraoperative PCI. However, the extent of peritoneal disease and the probability to achieve a complete macroscopic cytoreduction (CC-0/1) define the oncological benefit of the combined treatment concept. Thus, exploration along the round ligament of the liver with dissection of the parenchymatic bridge between liver segments III and IVb (pont hépatique) [14] and exploration of the omental bursa are mandatory. Beyond the technical feasibility of resection, histology, primary tumor, operative risk, pre- and postoperative quality of life, and patient preference have to be considered (fig. 1).

The greater omentum is often infiltrated by tumor nodules and may appear as 'omental cake' (fig. 2). Moreover, it has been shown to be a frequent area of intra-abdominal disease recurrence in different tumor entities. Thus, surgical resection should start with infragastric greater omentectomy including 


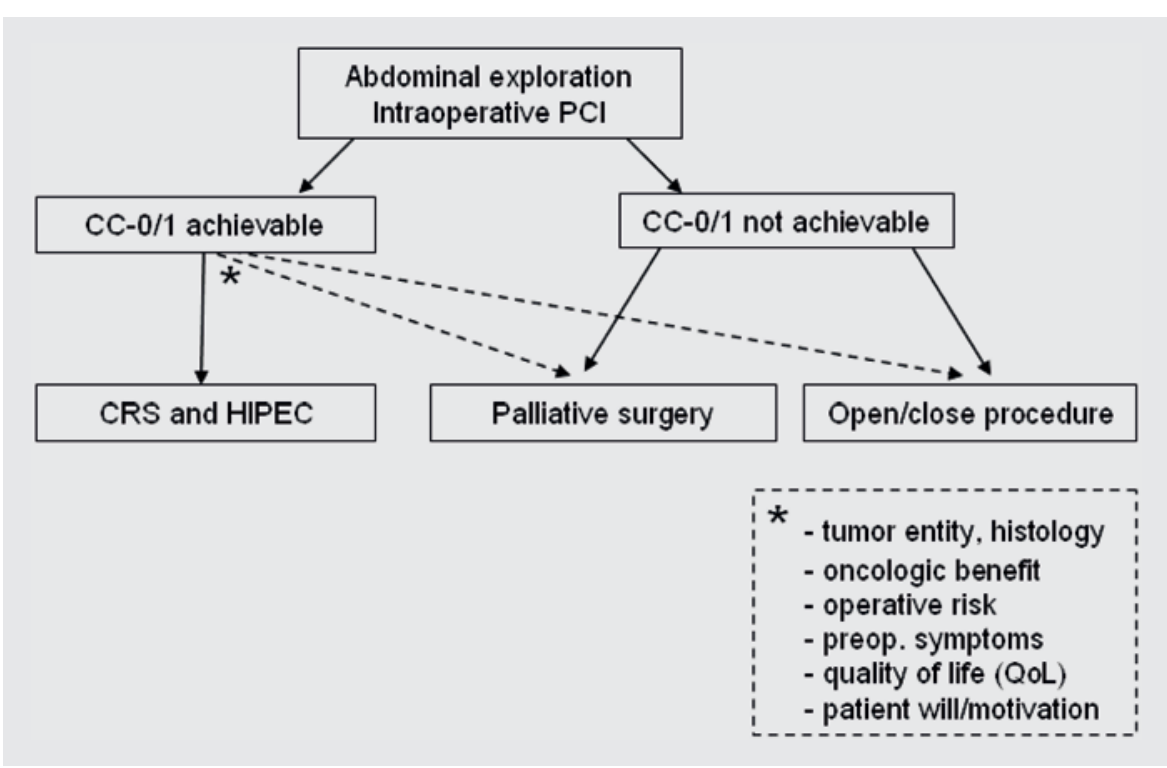

making.
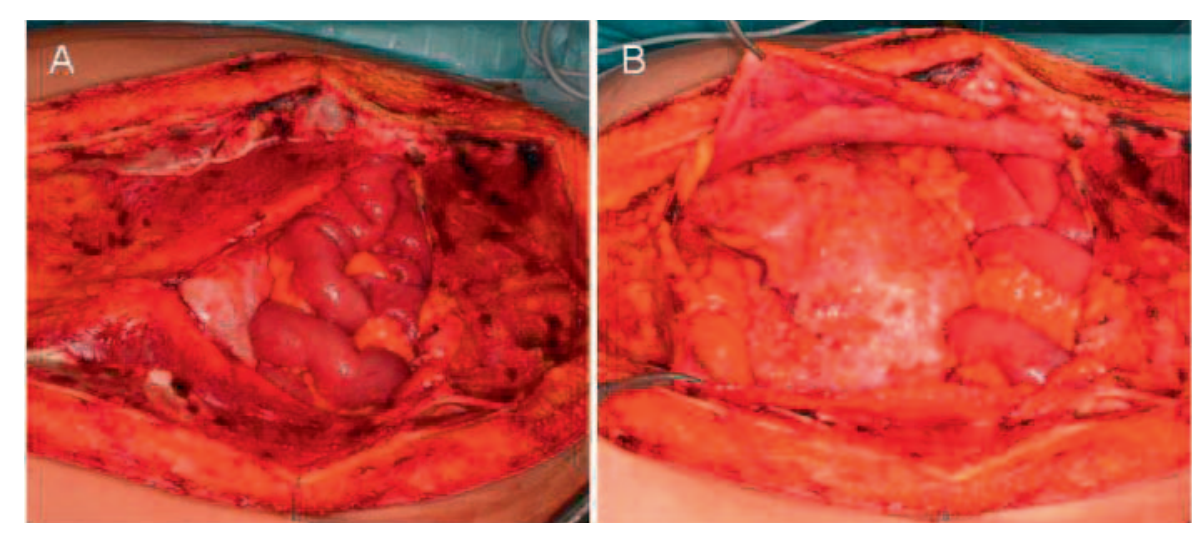

Fig. 2. 'Omental cake' of colorectal origin: tumor infiltration of the greater omentum in a patient with peritoneal metastasis from colorectal cancer $\mathbf{A}$ after partial incision (peritoneal window) and $\mathbf{B}$ after complete incision of the parietal peritoneum.

resection of the right gastroepiploic vessels. This step may free the abdomen from extensive tumor mass at the beginning of the operation and also allows for easier exploration and, if necessary, stripping of the omental bursa.

\section{Right and Left Upper Quadrant}

In the case of peritoneal metastasis affecting the right upper quadrant, the peritoneum must be stripped from beneath the right hemidiaphragm. Because tumor infiltration of the Glisson's capsule may require additional liver capsule resection, this procedure should be performed at the beginning to avoid an increasing risk of bleeding by swelling of the liver during a protracted CRS. The right liver lobe should be completely mobilized and the liver hilus should be exposed to allow for hepatic bleeding control if necessary. Peritoneal stripping is performed in four steps: i) incision of the peritoneum and separation of the right upper quadrant; ii) traction of the liver and stripping of the right diaphragm; iii) identification and preservation of the superior caval vein; and iv) transsection along the inferior caval vein, right colonic flexure, and duodenum. A cholecystectomy is often necessary due to tumor nodules on the peritoneum of the gall bladder and allows for easier access to the right subhepatic space (Morrison's pouch). In the case of tumor infiltration of the diaphragm, en bloc resection with consecutive suture of the diaphragm may be necessary. In these cases, intraoperative placement of a chest tube may help to avoid postoperative respiratory problems caused by pleural effusion. Additional hepatobiliary procedures such as extensive resection of the Glisson's capsule, liver resection, or bile duct resection may be necessary to obtain complete macroscopic cytoreduction and do not increase morbidity and mortality rates in experienced centers [15].

Peritonectomy of the left upper quadrant may be performed with or without splenectomy depending on the distribution pattern of the peritoneal implants. Thus, accurate initial exploration of the spleen is crucial to avoid residual tumor nodules in the left upper quadrant. The peritoneum is stripped from beneath the left diaphragm. If splenectomy is necessary, the spleen is mobilized via a lateral extraperitoneal approach and resected en bloc with the peritoneum of the left upper quadrant. Injury of the pancreatic tail should be avoided. 


\section{Lesser Omentum and Omental Bursa}

A failure analysis in 42 out of 118 patients with pseudomyxoma peritonei published by Zoetmulder et al. [16] showed that the most frequent site of recurrence after CRS and HIPEC is the subhepatic and lesser omentum area (28\%). Thus, an accurate exploration and surgical resection technique in this area plays a pivotal role regarding disease recurrence and oncological outcome of the patients. Since the right gastroepiploic vessels will have been ligated and resected during greater omentectomy, the left gastric artery should be preserved during lesser omentectomy to avoid gastrectomy due to iatrogenic ischemia. However, in patients with gastric cancer or gastric tumor infiltration of other origin, total gastrectomy might be performed in order to achieve a complete macroscopic cytoreduction while considering the postoperative impairment of quality of life. In many cases, partial gastrectomy, especially antrectomy, is sufficient for complete removal of all visible tumor nodules. Piso et al. [17] demonstrated no leakages related to gastric resections during CRS and HIPEC and acceptable postoperative morbidity in $37 \mathrm{pa}-$ tients. In some cases, an atypical left hepatic artery may arise from the left gastric artery that should be identified and preserved.

\section{Small Pelvis}

The small pelvis is frequently affected by peritoneal tumor implants. Moreover, the primary tumor such as ovarian cancer, sigmoid or rectal cancer, or in some cases appendiceal cancer may be located in or near to the small pelvis. Thus, peritonectomy of the small pelvis is a common procedure during CRS. The first step of pelvic peritonectomy is stripping the peritoneum from the posterior bladder wall. The urachus may be used as leading structure to start the peritonectomy. In the case of infiltration of the bladder, the affected part of the posterior wall should be resected and consecutively closed by interrupted sutures. When stripping the lateral wall of the small pelvis, the ureter has to be identified, mobilized, and preserved on both sides. In women, hysterectomy is often required to achieve complete macroscopic cytoreduction. For complete pelvic peritonectomy, the peritoneum is resected en bloc with the rectum including the Douglas pouch. Nevertheless, depending on the primary tumor and the dissemination pattern of tumor nodules, a Douglas pouch resection with rectal preservation may be performed.

In the case of peritoneal metastasis from ovarian cancer, ovariectomy with central ligation of the ovarian vessels, hysterectomy, and interaortocaval and iliac lymph node dissection are recommended [18]. Evers et al. [19] could show that at least $52 \%$ of patients with confirmed peritoneal metastases of colorectal or appendiceal origin have synchronous ovarian metastases. Based on these findings, ovariectomy should also be performed in women with peritoneal metastasis of colorectal or appendiceal origin [19]. However, as reported in 5 patients with pseudomyxoma peritonei and 2 patients with peri- toneal mesothelioma, childbearing after CRS and HIPEC is possible [20]. Thus, this issue has to be conscientiously discussed with fertile female patients before surgery.

\section{Hyperthermic Intraperitoneal Chemotherapy}

As a second step of the multimodal treatment concept, HIPEC is performed after complete macroscopic cytoreduction (CC-0/1). The goal of this treatment is to destroy residual tumor cells by the additive effects of high local doses of cytostatics and hyperthermia which has been shown to improve tissue penetration of different cytostatic agents and may lead to direct cytotoxic effects $[21,22]$. In the standard technique described by Paul H. Sugarbaker in 1995 [9], four drains are inserted intraperitoneally. The inflow drain is placed on the liver surface and three outflow drains are placed in the right and left subdiaphragmatic space and in the small pelvis, respectively. Depending on the used HIPEC device and drainage set, the number of drains may vary. The perfusate is applied via a heating and circulating pump system, and the temperature is monitored during the procedure. The intraperitoneal temperature should reach $41-42{ }^{\circ} \mathrm{C}$. Perfusion can be performed in an open, semi-open, or closed technique, depending on the standard operating procedures of the specialized center. The theoretical advantage of the open technique is a better control of the circulation and uniform distribution of the cytostatic agents. An important disadvantage might be the increased risk of contamination compared to the closed abdomen technique. Although a comparison of the existing studies is difficult, there seem to be no significant differences between the two techniques regarding morbidity and mortality rates as well as patient survival [23]. The safety of HIPEC for the therapist and the assisting personnel has been proven $[24,25]$.

In the case of diaphragmatic tumor infiltration with consecutive diaphragmatic resection during CRS, additional perfusion of the thorax might be discussed. The diaphragm could be left open and reconstructed after HIPEC. A chest tube might be integrated in the perfusion cycle. This procedure is also applicable in the case of perfusate loss during HIPEC due to insufficient primary suture of a diaphragmatic lesion. However, there is no consistent data showing a higher risk of local recurrence after primary closure of the defect without thoracic perfusion.

The specific HIPEC protocol depends on the tumor entity and the standard operating procedures of the specialized center. To date, the cytostatic agents, combinations and concentrations, as well as perfusion times for HIPEC, are not well standardized. Thus, numerous different combinations of cytostatic agents are used. Perfusion times range from 30 to $120 \mathrm{~min}$ [26]. Based on promising survival data for colorectal cancer, bidirectional oxaliplatin-based protocols that consist of intravenous application of 5-fluorouracil (5-FU) and folinic 
Table 1. Current HIPEC protocols of the University Medical Center Regensburg based on different tumor entities; protocols may be modified due to individual patient factors, and as there is no standardization and no consistent data, other HIPEC protocols may be used following the standard operating procedures (SOP) of the treating peritoneal cancer center

\begin{tabular}{llcccc}
\hline Entity & Agent & Dose, $\mathrm{mg} / \mathrm{m}^{2}$ & IP & IV & Perfusion \\
\hline CRC & 5-FU & 400 & & X & \\
Appendiceal cancer & folinic acid & 20 & & X & 30 min \\
PMP & oxaliplatin & 300 & X & & \\
\hline Gastric cancer & cisplatin & 75 & X & & \\
Ovarian cancer & doxorubicin & 15 & X & & 60 min \\
Peritoneal mesothelioma & & & & & \\
\hline
\end{tabular}

IP = Intraperitoneal; IV = intravenous; $\mathrm{CRC}=$ colorectal cancer, $\mathrm{PMP}=$ pseudomyxoma peritonei. acid prior to intraperitoneal application of heated oxaliplatin are increasingly used $[27,28]$. Initial data suggest that the intensified protocol does not increase perioperative morbidity [29]. However, evidence from prospective randomized trials is missing. The HIPEC protocol should be chosen on an individual basis considering tumor entity, response to previous chemotherapy regimens, and patient factors such as co-morbidities. The current standard HIPEC protocols of the University Medical Center Regensburg are summarized in table 1. Nevertheless, as long as consistent data from randomized controlled trials or comparative studies are missing, specific HIPEC protocols may not be generally recommended.

\section{Conclusion}

CRS and HIPEC should be considered as an efficient therapeutic option for selected patients with peritoneal surface malignancies within an interdisciplinary treatment concept. The multimodal approach can be performed with acceptable morbidity and low mortality in specialized centers. Nevertheless, due to long learning curves, consistent surgical tutoring and training is strongly recommended. Several technical aspects as well as oncological and surgical outcome and individual patient factors should be taken into account before and during surgery.

\section{Disclosure Statement}

The authors have no competing interests to declare.

\section{References}

1 Glockzin G, Schlitt HJ, Piso P: Peritoneal carcinomatosis: patients selection, perioperative complications and quality of life related to cytoreductive surgery and hyperthermic intraperitoneal chemotherapy. World J Surg Oncol 2009;7:5.

-2 Sadeghi B, Arvieux C, Glehen O, Beaujard AC, Rivoire M, Baulieux J, Fontaumard E, Brachet A, Caillot JL, Faure JL, Porcheron J, Peix JL, Francois Y, Vignal J, Gilly FN: Peritoneal carcinomatosis from non-gynecologic malignancies: results of the EVOCAPE 1 multicentric prospective study. Cancer 2000;88:358-363.

3 Sugarbaker PH, Cunliffe WJ, Belliveau J, de Bruijn EA, Graves T, Mullins RE, Schlag P: Rationale for integrating early postoperative intraperitoneal chemotherapy into the surgical treatment of gastrointestinal cancer. Semin Oncol 1989;16:83-97.

4 Sugarbaker PH: New standard of care for appendiceal epithelial neoplasms and pseudomyxoma peritonei syndrome? Lancet Oncol 2006;7:69-76.

$\checkmark 5$ Glehen O, Gilly FN, Boutitie F, Bereder JM, Quenet F, Sideris L, Mansvelt B, Lorimier G, Msika S, Elias D: Toward curative treatment of peritoneal carcinomatosis from nonovarian origin by cytoreductive surgery combined with perioperative intraperitoneal chemotherapy: a multi-institutional study of 1,290 patients. Cancer 2010;116: 5608-5618.
6 Chua TC, Yan TD, Saxena A, Morris DL: Should the treatment of peritoneal carcinomatosis by cytoreductive surgery and hyperthermic intraperitoneal chemotherapy still be regarded as a highly morbid procedure? A systematic review of morbidity and mortality. Ann Surg 2009;249:900-907.

7 Moran BJ, Mukherjee A, Sexton R: Operability and early outcome in 100 consecutive laparotomies for peritoneal malignancy. Br J Surg 2006;93:100104.

8 Glehen O, Gilly FN: Quantitative prognostic indicators of peritoneal surface malignancy: carcinomatosis, sarcomatosis, and peritoneal mesothelioma. Surg Oncol Clin N Am 2003;12:649-671.

$\checkmark 9$ Sugarbaker PH: Peritonectomy procedures. Ann Surg 1995;221:29-42.

10 Kusamura S, Baratti D, Virzi S, Bonomi S, Iusco DR, Grassi A, Hutanu I, Deraco M: Learning curve for cytoreductive surgery and hyperthermic intraperitoneal chemotherapy in peritoneal surface malignancies: analysis of two centres. J Surg Oncol 2013;107:312-319.

11 Jacquet P, Sugarbaker PH: Clinical research methodologies in diagnosis and staging of patients with peritoneal carcinomatosis. Cancer Treat Res 1996; 82:359-374.
2 Esquivel J, Chua TC, Stojadinovic A, Melero JT, Levine EA, Gutman M, Howard R, Piso P, Nissan A, Gomez-Portilla A, Gonzalez-Bayon L, Gonzalez-Moreno S, Shen P, Stewart JH, Sugarbaker PH, Barone RM, Hoefer R, Morris DL, Sardi A, Sticca RP: Accuracy and clinical relevance of computed tomography scan interpretation of peritoneal cancer index in colorectal cancer peritoneal carcinomatosis: a multi-institutional study. J Surg Oncol 2010;102:565-570.

13 Esquivel J, Chua TC: CT versus intraoperative peritoneal cancer index in colorectal cancer peritoneal carcinomatosis: importance of the difference between statistical significance and clinical relevance. Ann Surg Oncol 2009;16:2662-2663.

14 Sugarbaker PH: Pont hepatique (hepatic bridge), an important anatomic structure in cytoreductive surgery. J Surg Oncol 2010;101:251-252.

15 Glockzin G, Renner P, Popp FC, Dahlke MH, von Breitenbuch P, Schlitt HJ, Piso P: Hepatobiliary procedures in patients undergoing cytoreductive surgery and hyperthermic intraperitoneal chemotherapy. Ann Surg Oncol 2011;18:1052-1059.

16 Zoetmulder FA, Sugarbaker PH: Patterns of failure following treatment of pseudomyxoma peritonei of appendiceal origin. Eur J Cancer 1996; 32A:1727-1733. 
17 Piso P, Slowik P, Popp F, Dahlke MH, Glockzin G, Schlitt HJ: Safety of gastric resections during cytoreductive surgery and hyperthermic intraperitoneal chemotherapy for peritoneal carcinomatosis. Ann Surg Oncol 2009;16:2188-2194.

-18 Morgan RJ Jr, Alvarez RD, Armstrong DK, Burger RA, Castells M, Chen LM, Copeland L, Crispens MA, Gershenson D, Gray H, Hakam A, Havrilesky LJ, Johnston C, Lele S, Martin L, Matulonis UA, O'Malley DM, Penson RT, Remmenga SW, Sabbatini P, Santoso JT, Schilder RJ, Schink J, Teng N, Werner TL, Hughes M, Dwyer MA: Ovarian cancer, version 3, 2012. J Natl Compr Canc Netw 2012;10:1339-1349.

19 Evers DJ, Verwaal VJ: Indication for oophorectomy during cytoreduction for intraperitoneal metastatic spread of colorectal or appendiceal origin. Br J Surg 2011;98:287-292.

20 Ortega-Deballon P, Glehen O, Levine E, Piso P, Sugarbaker PH, Hayes-Jordan A, Facy A, Bakrin N, Rat P: Childbearing after hyperthermic intraperitoneal chemotherapy: results from an international survey. Ann Surg Oncol 2011;18:2297-2301.

21 Ceelen WP, Flessner MF: Intraperitoneal therapy for peritoneal tumors: biophysics and clinical evidence. Nat Rev Clin Oncol 2010;7:108-115.
22 Sugarbaker PH: Laboratory and clinical basis for hyperthermia as a component of intracavitary chemotherapy. Int J Hyperthermia 2007;23:431-442.

23 Glehen O, Cotte E, Kusamura S, Deraco M, Baratti D, Passot G, Beaujard AC, Noel GF: Hyperthermic intraperitoneal chemotherapy: nomenclature and modalities of perfusion. J Surg Oncol 2008;98:242-246.

24 Stuart OA, Stephens AD, Welch L, Sugarbaker PH: Safety monitoring of the coliseum technique for heated intraoperative intraperitoneal chemotherapy with mitomycin C. Ann Surg Oncol 2002;9: 186-191.

25 Naslund Andreasson S, Anundi H, Thoren SB, Ehrsson H, Mahteme H: Is platinum present in blood and urine from treatment givers during hyperthermic intraperitoneal chemotherapy? J Oncol 2010;2010:649719.

26 Gonzalez-Moreno S, Gonzalez-Bayon LA, OrtegaPerez G: Hyperthermic intraperitoneal chemotherapy: rationale and technique. World J Gastrointest Oncol 2010;2:68-75.
27 Hompes D, D'Hoore A, Van Cutsem E, Fieuws S, Ceelen W, Peeters M, Van der Speeten K, Bertrand C, Legendre H, Kerger J: The treatment of peritoneal carcinomatosis of colorectal cancer with complete cytoreductive surgery and hyperthermic intraperitoneal peroperative chemotherapy (HIPEC) with oxaliplatin: a Belgian multicentre prospective phase II clinical study. Ann Surg Oncol 2012;19:2186-2194.

28 Elias D, Lefevre JH, Chevalier J, Brouquet A, Marchal F, Classe JM, Ferron G, Guilloit JM, Meeus P, Goere D, Bonastre J: Complete cytoreductive surgery plus intraperitoneal chemohyperthermia with oxaliplatin for peritoneal carcinomatosis of colorectal origin. J Clin Oncol 2009;27:681-685.

29 Glockzin G, von Breitenbuch P, Schlitt HJ, Piso P: Treatment-related morbidity and toxicity of CRS and oxaliplatin-based HIPEC compared to a mitomycin and doxorubicin-based HIPEC protocol in patients with peritoneal carcinomatosis: a matchedpair analysis. J Surg Oncol 2013;107:574-582. 